\title{
A Method for the Correction of EBSPs Distorted by Lens Magnetic Fields
}

\author{
C.T.Chou ${ }^{1}$, K. Thomsen ${ }^{2}$, J Goulden ${ }^{1}$, H. Jiang ${ }^{1}$, \\ 1 Oxford Instruments NanoAnalysis, Halifax Road, High Wycombe, HP12 3SE, UK \\ 2 Oxford Instruments Consultant
}

EBSD analysis can be impaired by the presence of a magnetic field introduced by the immersion lenses used in some SEM's to give improved image resolution. In these SEMs the final lens for focusing the electron beam produces a magnetic field, and typically the sample is immersed in this lens field, allowing tighter focus of the electron beam and offering improved image resolution. However, this magnetic field distorts and shifts the electron back-scattered patterns (EBSPs). As a result straight features, such as Kikuchi bands, appear not only curved, but are also shifted.

Band recognition in distorted patterns is difficult and the shift of the pattern causes the calibrated pattern centre position, determined without a magnetic field, to be erroneous. These difficulties confound correct and accurate indexing of EBSP's.

A method is proposed to correct for the distortion in EBSPs. This technique uses a dipole model to describe the distortion and an image correlation is used to find the strength of the magnetic field. To perform the correction requires a pair of undistorted and distorted patterns from the same crystal or grain. These patterns are used to determine the size of the magnetic field. The model is optimized by varying the dipole field strength parameter until the correlation between the corrected and undistorted pattern is maximized. This optimized model is then applied to all subsequent patterns

This method enables the correction of distorted EBSD patterns, with an accurate determination of band and pattern centre position. Thus, patterns collected in the presence of a magnetic field, where the SEM has been optimized for high resolution imaging, can still be correctly indexed.

A series of EBSPs are shown in Figures 1a-e. These patterns were collected from a silicon sample. Figure $1 \mathrm{a}$ and $1 \mathrm{~b}$ are the undistorted pattern. Figure 1c shows the distortion of the Kikuchi bands resulting from the immersion lens magnetic field. The application of the dipole model correction (figure 1d and 1e) delivers an EBSP which can be successfully indexed. 


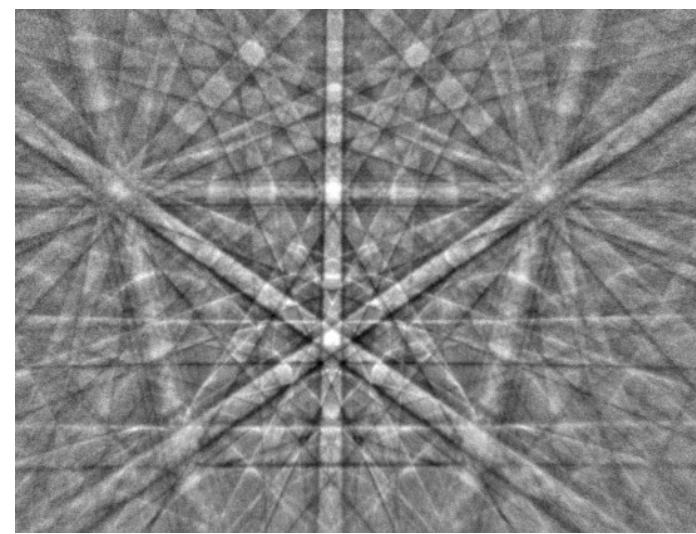

Figure 1a: A normal undistorted EBSP from silicon

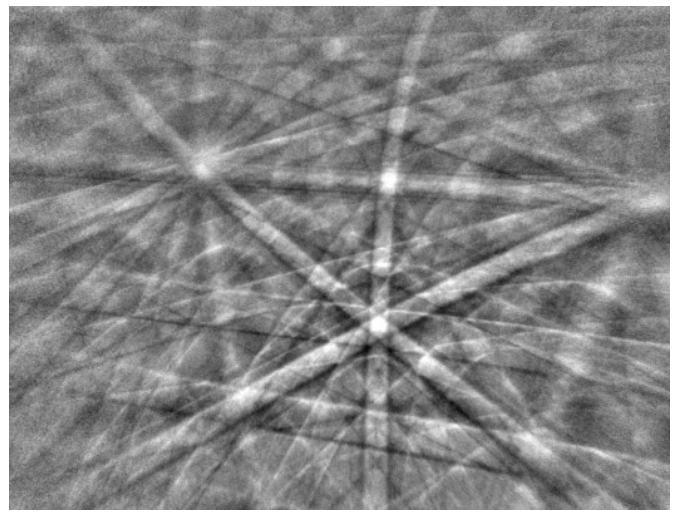

Figure 1c: Distorted EBSP from the same sample in a immersion lens

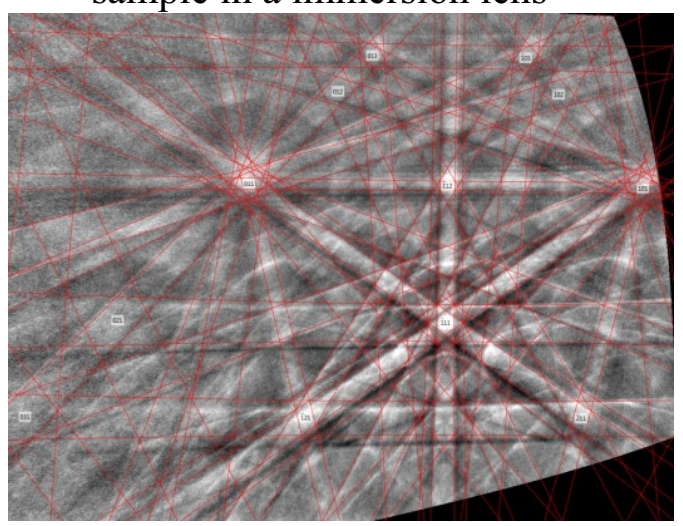

Figure 1e: Indexing of the corrected EBSP

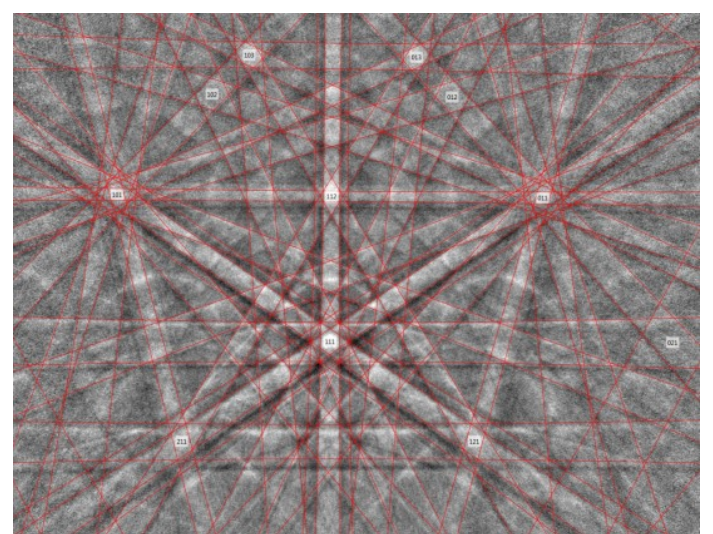

Figure 1b: Indexing of EBSP in figure 1a

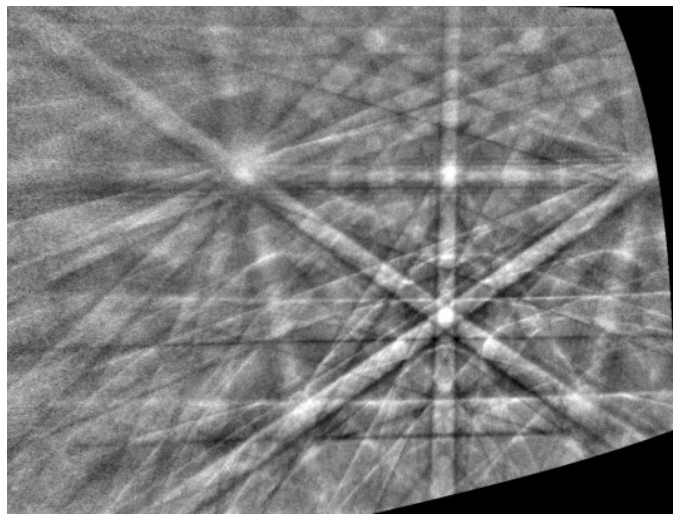

Figure 1d: Correction of the distorted EBSP 